\title{
Teaching Cases of the Month
}

\section{Hematologic Abnormalities Associated With Lung Carcinoma}

\author{
Majid Shafiq MD and Saiprakash Venkateshiah MD
}

\section{Introduction}

Leukocytosis, thrombocytosis, and anemia are often associated with lung cancer, and each can be a source or marker of morbidity or mortality. We report a patient with metastatic adenocarcinoma of the lung that was associated with leukocytosis, thrombocytosis, and anemia. In our patient, initiation of chest radiotherapy markedly improved his symptoms and hematology panel.

\section{Case Summary}

At the Atlanta Veterans Affairs Medical Center, we saw a 60-year-old white male with an 80-pack-year history of smoking, mild COPD, and stage IV adenocarcinoma of the lung (diagnosed 4 months prior to this presentation). He presented to the emergency room after 2 weeks of worsening dyspnea and cough productive of yellow sputum. His wife also reported that his face and neck had become swollen over the past several weeks. He did not report fevers, chills, or any other complaints.

Approximately 3 months before this presentation, he had undergone tracheal stenting for airway compromise caused by the impinging tumor. This was followed by chest radiotherapy (30 Gy in 12 fractions) over 14 days. At that time his white blood cell (WBC) count had decreased from $12.2 \mathrm{~K}$ cells $/ \mathrm{mL}$ at the start of radiotherapy to $5.7 \mathrm{~K}$ cells $/ \mathrm{mL}$ at the end. The hemoglobin and platelet counts were normal at the beginning of radiotherapy and had remained relatively unchanged afterwards. A computed tomogram 14 days after the conclusion of radiotherapy showed a slight reduction in tumor burden, but further

\footnotetext{
Majid Shafiq MD and Saiprakash Venkateshiah MD are affiliated with the Department of Internal Medicine, Emory University School of Medicine, Atlanta, Georgia. Saiprakash Venkateshiah MD is also affiliated with the Veterans Affairs Medical Center, Decatur, Georgia.

The authors have disclosed no conflicts of interest.
}

Correspondence: Majid Shafiq MD, Department of Internal Medicine, Emory University School of Medicine, 69 Jesse Hill Jr Drive SE, Atlanta GA 30303. E-mail: mshafiq@emory.edu.

DOI: $10.4187 /$ respcare.00944 imaging studies over the next 2 months showed rapid progression. One month before this presentation he was started on palliative chemotherapy with gemcitabine and carboplatin. The plan was to continue palliative chemotherapy and consider future radiotherapy in another 3 months' time if he responded favorably to the former.

On physical examination, he was afebrile (maximum temperature $\left.37.4^{\circ} \mathrm{C}\right)$, normotensive $(122 / 72 \mathrm{~mm} \mathrm{Hg}$ ), mildly dyspneic, and had a respiratory rate of 22 breaths/ min. He also had mild swelling and erythema of the face and neck, and blanching telangiectasias on the upper chest and back. Chest radiograph revealed a new right-upperlobe infiltrate and a possible associated cavitary lesion (Fig. 1). Computed tomogram confirmed the new cavitary lesion, which had a thick-walled border, thought to represent fibrosis and air-space consolidation secondary to radiation, rather than tumor progression. A persistent middle mediastinal and right-hilar mass and substantial narrowing of the superior vena cava were also visualized (Fig. 2). The tracheal stent was in place, and there was very mild tracheal narrowing distal to the stent (Fig. 3).

Three weeks prior to the current presentation, his WBC count had been $4,000 \mathrm{~K}$ cells $/ \mathrm{mL}$, platelet count $258 \mathrm{~K}$ cells/ $\mathrm{mL}$, hemoglobin $8.8 \mathrm{~g} / \mathrm{dL}$, and mean corpuscular volume $88.7 \mathrm{fL}$. At current presentation his WBC count was $39,500 \mathrm{~K}$ cells $/ \mathrm{mL}$ (73\% segmented neutrophils, $6 \%$ lymphocytes, $12 \%$ monocytes), platelets $942 \mathrm{~K}$ cells $/ \mathrm{mL}$, hemoglobin $7.5 \mathrm{~g} / \mathrm{dL}$, and mean corpuscular volume $87.8 \mathrm{fL}$. The chemistry panel was within normal limits, and culture results (both sputum and blood) remained negative.

A venogram demonstrated a non-occlusive thrombus in the superior vena cava, and substantial tumor impingement on the vein. He underwent thrombectomy followed by angioplasty of the superior vena cava. He started another round of chest radiotherapy (30 Gy over 12 fractions) and his palliative chemotherapy was discontinued.

Subsequent to admission, he also received an 8-day course of vancomycin, piperacillin-tazobactam, and levofloxacin for empirical treatment of healthcare-associated pneumonia (since his last hospital discharge had occurred less than 90 days prior to the current presentation). However, the first 6 days of this treatment failed to bring any substantial symptom improvement or an appreciable change 


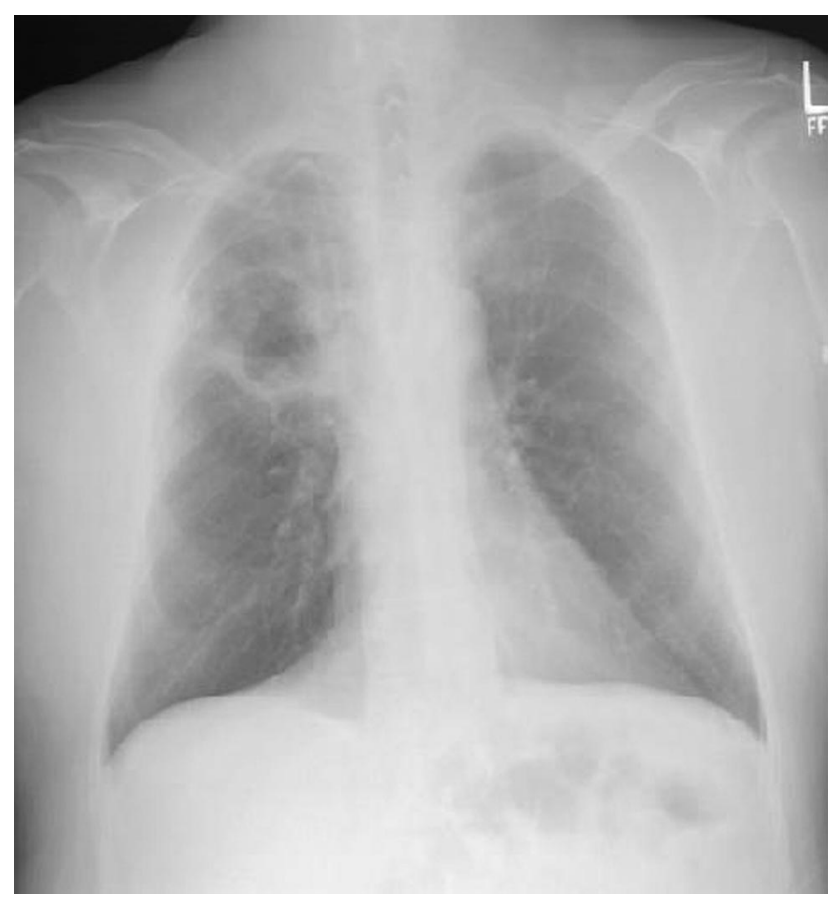

Fig. 1. Frontal chest radiograph shows a new right-upper-lobe infiltrate, a possible associated cavitary lesion, a portacath (on the right-side), the tip of which is projecting toward the superior vena cava, and a stable tracheal stent.

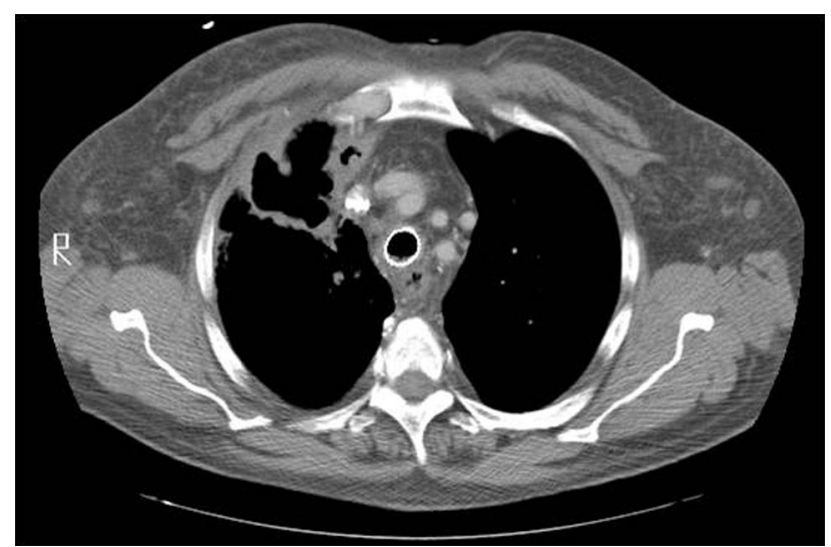

Fig. 2. Computed tomogram (mediastinal window) shows substantial narrowing of the superior vena cava, caused by a middle mediastinal and right hilar mass.

in the hematology panel. On the other hand, soon after radiation therapy was instituted (on day 6 of admission), both symptoms and the hematology panel began to improve.

During his first hospital week he received 6 units of packed red cells, as his hematocrit had continued to steadily decline. The peripheral blood smear was normal except for mild anisocytosis and poikilocytosis, whereas the reticulocyte proliferation index was low-normal (suggesting a hypoproliferative cause of the anemia). Iron studies, per-

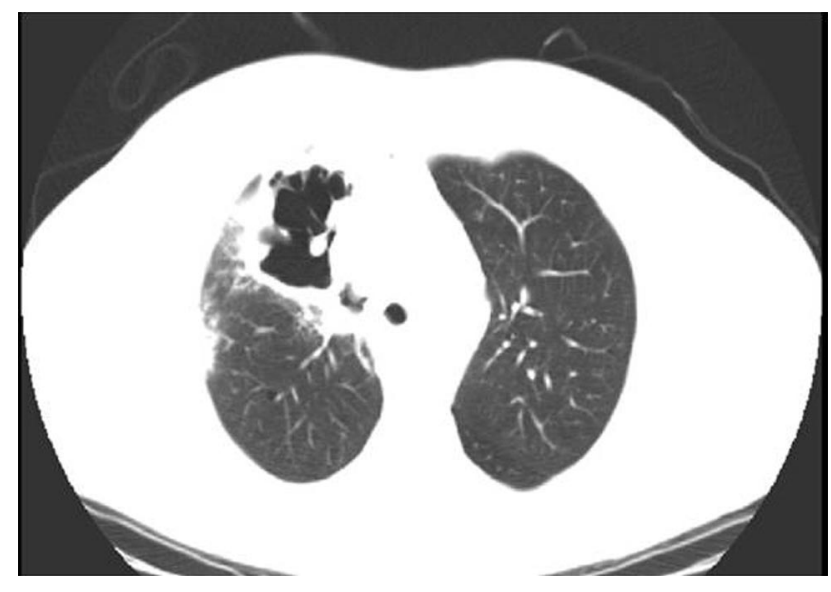

Fig. 3. Computed tomogram (lung window) shows a large cavitary lesion in the right upper lobe, with a thick-walled border.

formed at this time, pointed towards anemia of chronic disease (notwithstanding the caveat that ferritin levels may have limited diagnostic value during acute inflammation). During the second week, soon after having commenced radiotherapy, his hematocrit stabilized and he did not require any more blood products.

On the 14th hospital day his WBC count was $12.5 \mathrm{~K}$ cells/ $\mathrm{mL}$, his hemoglobin was $10.6 \mathrm{~g} / \mathrm{dL}$, and his platelet count was $590 \mathrm{~K}$ cells $/ \mathrm{mL}$.

His dyspnea improved slightly in response to care during the first 6 days (including high-flow oxygen, frequent nebulized bronchodilators, and broad-spectrum antibiotics), but his productive cough remained unchanged. By day 2 of radiotherapy, however, his cough had improved substantially and he also reported feeling much less dyspneic than before.

\section{Discussion}

Certain hematologic abnormalities can be independently associated with a poor prognosis in lung cancer. Leukocytosis, anemia, and thrombocytosis have all been reported in patients with solid tumors, chiefly lung cancer and colorectal cancer. ${ }^{1}$ Various studies have evaluated the clinical impact and prognostic value of these abnormal blood cell counts in lung cancer. The association of leukocytosis, anemia, and thrombocytosis with lung cancer is not uncommon. A study with 1,201 newly diagnosed lung cancer patients found anemia, leukocytosis, and thrombocytosis in $56 \%, 32 \%$, and $17 \%$ of the patients, respectively. ${ }^{2}$

\section{Leukocytosis}

A high leukocyte count, especially with a predominance of granulocytes, is often presumed to be secondary to a bacterial infection. However, other causes, including ad- 
vanced malignancy, hemorrhage, and corticosteroid use, must also be considered. Fever can be a useful differentiating factor, presenting more commonly in patients with infection. Notably, infection is less likely to be the cause of marked leukocytosis in a patient afflicted with a solid malignancy. Granger et al studied 758 solid-tumor patients with extreme leukocytosis $(>40,000 \mathrm{~K} / \mu \mathrm{L})$. Recent administration of hematopoietic growth factors was the most common etiology (69\%); infection was a distant second $(15 \%)$. Other common causes included a paraneoplastic leukemoid reaction $(10 \%)$ and high-dose corticosteroids (5\%). Even though infection was a relatively uncommon cause, the leukocytosis was almost always dominated by granulocytes (as in our patient). ${ }^{3}$

In our patient, the most likely etiology of his leukocytosis was a paraneoplastic leukemoid reaction. He had not received any colony-stimulating factors in the preceding few weeks. An infectious etiology is less likely, given that he did not have a fever and his cell counts hardly changed during several days of broad-spectrum antibiotic therapy. He had not received any corticosteroids recently, nor did he turn out to have a new leukemia. This kind of a paraneoplastic leukemoid reaction is thought to be most commonly seen with lung cancer (chiefly non-small-cell), as in our patient, and colorectal cancer. It is thought to be due to the production of hematopoietic growth factors by tumor cells. ${ }^{1}$

A diagnosis of paraneoplastic leukemoid reaction (also referred to as tumor-related leukocytosis) is important, because it has substantial prognostic value. There have been a few case reports of patients achieving resolution of their malignancy-related leukocytosis with the initiation of antineoplastic therapy. ${ }^{4}$ For the most part, however, these abnormalities persist and these patients tend to have poor outcomes. In a prospective study by Kasuga et al, newly diagnosed lung-cancer patients had a median survival of 4.6 months if they developed tumor-related leukocytosis (range 11,400-190,000 K cells/ $\mu \mathrm{L}$ ). ${ }^{5}$ Those with no tumor-related leukocytosis had a median survival of 20.8 months, whereas those with infection-related leukocytosis had a median survival of 15.5 months. The difference in survival between the tumor-related leukocytosis subset and each of the other subsets was statistically significant. Notably, most of the cases of tumor-related leukocytosis were marked by a predominance of granulocytes.

Besides the cancer itself, chemotherapy can also be responsible for leukocytosis. Lissoni and others reported that lung-cancer patients who were treated with gemcitabine and cisplatin were significantly more likely to develop leukocytosis. ${ }^{6}$ Our patient did get one cycle of gemcitabine and cisplatin starting one month prior to presentation. However, Lissoni et al reported an increase in lymphocytosis, whereas our patient had granulocytosis.

\section{Anemia}

Like leukocytosis, anemia has also been reported in association with lung cancer. Studies have reported its incidence as $50-60 \%,{ }^{7}$ which is much higher than that seen in colorectal and breast cancers (around 10-20\% each). Anemia of chronic disease is believed to be the major underlying cause of anemia in cancer patients. However, platinum-based chemotherapy is also an important factor and may at least partially explain why lung cancer has one of the highest rates of anemia among the common malignancies. Either anemia of chronic disease or platinumbased chemotherapy — or both — could have been responsible for our patient's anemia.

Anemia in the context of a malignancy predicts a poor outcome. ${ }^{7}$ It is associated with fatigue in 3 of every 4 cancer patients, as measured with the general version of the Functional Assessment of Cancer Therapy (FACT-G) questionnaire, and decreases quality of life. ${ }^{8}$ In a telephone survey of 419 patients, $61 \%$ of cancer patients said fatigue affected their daily lives more than pain. ${ }^{9}$

Anemia is also associated with a higher cancer recurrence rate and shorter survival after radiotherapy. This may reflect more aggressive or extensive tumor burden, rather than a direct effect of the anemia itself, but there is some evidence that tumor-cell hypoxia, potentially exacerbated by anemia, may adversely affect radiosensitivity. However, to date only a weak association has been reported between anemia and tumor radiosensitivity. ${ }^{10} \mathrm{Ra}-$ diation therapy appeared to improve our patient's hematocrit, as he did not need any more blood products after the institution of radiotherapy.

\section{Thrombocytosis}

Thrombocytosis is also a relatively common laboratory abnormality in lung-cancer patients, with a prevalence of $16-46 \% .{ }^{11,12}$ Reactive thrombocytosis in lung cancer has been suggested to be part of the systemic inflammatory reaction for which interleukin-1 beta and interleukin-6 may be intermediate, though not independent, mediators. ${ }^{13}$ Thrombocytosis is an independent predictor of poor prognosis in lung-cancer patients. ${ }^{11,14}$ Curiously, though, it has not been associated with an increased incidence of venous thromboembolism.

\section{Other Hematologic Abnormalities}

Various other hematologic abnormalities have also been reported in association with lung cancer, including elevated eosinophil count in blood and in the pleural fluid. Interleukin-5 has been implicated as a causative factor. ${ }^{15}$ Cryoglobulinemia has also been known to accompany various solid tumors, including lung cancer. In 1961, Domz 
and Chapman reported a case of cryoglobulinemia and digital gangrene that resolved following surgical resection of a non-metastatic lung adenocarcinoma. ${ }^{16}$

Pulmonary tumor thrombotic microangiopathy is characterized by intimal proliferation of small pulmonary arteries and arterioles, and is believed to occur from tumoremboli-induced local activation of coagulation and fibrocellular intimal proliferation. Ultimately, it can lead to stenosis or occlusion and consequent pulmonary hypertension. The most commonly implicated underlying cause is metastatic gastric adenocarcinoma, but Uruga et al recently reported a case of pulmonary tumor thrombotic microangiopathy in association with adenocarcinoma of the lung. ${ }^{17}$

\section{Teaching Points}

- Anemia, thrombocytosis, and leukocytosis (often with granulocytosis) can all be seen in patients with lung cancer, sometimes simultaneously. While it is essential to consider the possibility of alternative etiologies for these abnormalities, it is also important to recognize these as common manifestations of the malignancy itself, which should help avoid unnecessarily extensive work-ups and their considerable morbidity and cost, and enable faster institution of anti-neoplastic therapy.

- Both tumor-related leukocytosis and tumor-related thrombocytosis have prognostic value, whereas anemia can have a weakly negative effect on tumor radiosensitivity.

- Chest radiation therapy may greatly improve these abnormalities, which may be secondary to a reduction of the tumor burden and, hence, its paraneoplastic effects.

\section{ACKNOWLEDGMENTS}

We thank Fawad M Shuaib MD, Department of Internal Medicine, Weiss Memorial Hospital, Chicago, Illinois, for his excellent help with the literature search.

\section{REFERENCES}

1. Shoenfeld Y, Tal A, Berliner S, Pinkhas J. Leukocytosis in nonhematological malignancies: a possible tumor-associated marker. J Cancer Res Clin Oncol 1986;111(1):54-58.
2. Ferrigno D, Buccheri G. Hematologic counts and clinical correlates in 1201 newly diagnosed lung cancer patients. Monaldi Arch Chest Dis 2003;59(3):193-198.

3. Granger JM, Kontoyiannis DP. Etiology and outcome of extreme leukocytosis in 758 nonhematologic cancer patients: a retrospective, single-institution study. Cancer 2009;115(17):3919-3923.

4. Qureshi KM, Raman AK, Tan D, Fakih MG. Leukemoid reaction in pancreatic cancer: a case report and review of the literature. JOP 2006;7(6):631-634.

5. Kasuga I, Makino S, Kiyokawa H, Katoh H, Ebihara Y, Ohyashiki $\mathrm{K}$. Tumor-related leukocytosis is linked with poor prognosis in patients with lung carcinoma. Cancer 2001;92(9):2399-2405.

6. Lissoni P, Fumagalli L, Brivio F, Rovelli F, Messina G, Di Fede G, et al. Cancer chemotherapy-induced lymphocytosis: a revolutionary discovery in the medical oncology. J Biol Regul Homeost Agents 2006;20(1-2):29-35.

7. Langer CJ, Choy H, Glaspy JA, Colowick A. Standards of care for anemia management in oncology: focus on lung carcinoma. Cancer 2002;95(3):613-623.

8. Cella D. Factors influencing quality of life in cancer patients: anemia and fatigue. Semin Oncol 1998;25(3 Suppl 7):43-46.

9. Vogelzang NJ, Breitbart W, Cella D. Patient, caregiver, and oncologist perceptions of cancer-related fatigue: results of a tripart assessment survey. Semin Hematol 1997;34(3 Suppl 2):4-12.

10. Brizel DM, Dodge RK, Clough RW, Dewhirst MW. Oxygenation of head and neck cancer: changes during radiotherapy and impact on treatment outcome. Radiother Oncol 1999;53(2):113-117.

11. Engan T, Hannisdal E. Blood analyses as prognostic factors in primary lung cancer. Acta Oncol 1990;29(2):151-154.

12. Gislason T, Nou E. Sedimentation rate, leucocytes, platelet count and haemoglobin in bronchial carcinoma: an epidemiological study. Eur J Respir Dis 1985;66(2):141-146.

13. Alexandrakis MG, Passam FH, Perisinakis K, Ganotakis E, Margantinis G, Kyriakou DS, Bouros D. Serum pro-inflammatory cytokines and its relationship to clinical parameters in lung cancer patients with reactive thrombocytosis. Respir Med 2002;96(8):553-558.

14. Aoe K, Hiraki A, Ueoka H, Kiura K, Tabata M, Tanaka M, Tanimoto $\mathrm{M}$. Thrombocytosis as a useful prognostic indicator in patients with lung cancer. Respiration 2004;71(2):170-173.

15. Pandit R, Scholnik A, Wulfekuhler L, Dimitrov N. Non-small-cell lung cancer associated with excessive eosinophilia and secretion of interleukin-5 as a paraneoplastic syndrome. Am J Hematol 2007; 82(3):234-237.

16. Domz CA, Chapman CG. Pseudo-Raynaud's: cryoglobulinemia secondary to occult neoplasm. Calif Med 1961;95(6):391-393.

17. Uruga H, Morokawa N, Enomoto T, Takaya H, Miyamoto A, Kishi $\mathrm{K}$, et al. [A case of pulmonary tumor thrombotic microangiopathy associated with lung adenocarcinoma diagnosed by CT-guided lung biopsy.] Nihon Kokyuki Gakkai Zasshi 2008;46(11):928-933. Article in Japanese. 